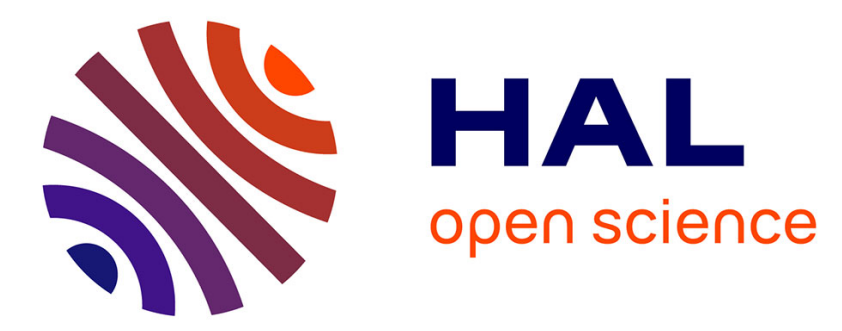

\title{
Expériences de justice et représentations sociales : l'exemple du non-recours aux droits
}

\author{
Arnaud Béal, Nikos Kalampalikis, Nicolas Fieulaine, Valérie Haas
}

\section{To cite this version:}

Arnaud Béal, Nikos Kalampalikis, Nicolas Fieulaine, Valérie Haas. Expériences de justice et représentations sociales: l'exemple du non-recours aux droits. Les cahiers Internationaux de Psychologie Sociale, 2014, 103 (3). halshs-01205959

\section{HAL Id: halshs-01205959 \\ https://shs.hal.science/halshs-01205959}

Submitted on 10 Jul 2017

HAL is a multi-disciplinary open access archive for the deposit and dissemination of scientific research documents, whether they are published or not. The documents may come from teaching and research institutions in France or abroad, or from public or private research centers.
L'archive ouverte pluridisciplinaire HAL, est destinée au dépôt et à la diffusion de documents scientifiques de niveau recherche, publiés ou non, émanant des établissements d'enseignement et de recherche français ou étrangers, des laboratoires publics ou privés. 


\section{Expériences de justice et représentations sociales : l'exemple du non-recours aux droits}

ARTICLE · JANUARY 2014

DOI: $10.3917 /$ cips.103.0549

CITATION

1

4 AUTHORS, INCLUDING:

\section{Nikos Kalampalikis}

Université Lumiere Lyon 2

71 PUBLICATIONS 216 CITATIONS

SEE PROFILE
Valérie Haas

Université Lumiere Lyon 2

18 PUBLICATIONS 40 CITATIONS

SEE PROFILE 


\title{
EXPÉRIENCES DE JUSTICE ET REPRÉSENTATIONS SOCIALES : L'EXEMPLE DU NON-RECOURS AUX DROITS
}

\author{
Arnaud Beal et al.
}

Presses universitaires de Liège | Les cahiers internationaux de psychologie sociale

\author{
2014/3 - Numéro 103 \\ pages 549 à 573
}

ISSN 0777-0707

Article disponible en ligne à l'adresse:

http://www.cairn.info/revue-les-cahiers-internationaux-de-psychologie-sociale-2014-3-page-549.htm

Pour citer cet article :

Beal Arnaud et al., « Expériences de justice et représentations sociales : l'exemple du non-recours aux droits », Les cahiers internationaux de psychologie sociale, 2014/3 Numéro 103, p. 549-573.

Distribution électronique Cairn.info pour Presses universitaires de Liège.

(c) Presses universitaires de Liège. Tous droits réservés pour tous pays.

La reproduction ou représentation de cet article, notamment par photocopie, n'est autorisée que dans les limites des conditions générales d'utilisation du site ou, le cas échéant, des conditions générales de la licence souscrite par votre établissement. Toute autre reproduction ou représentation, en tout ou partie, sous quelque forme et de quelque manière que ce soit, est interdite sauf accord préalable et écrit de l'éditeur, en dehors des cas prévus par la législation en vigueur en France. II est précisé que son stockage dans une base de données est également interdit. 


\section{$\mathbf{6 6}$ Expériences de justic e et représenta tions sociales: l'exemple du non-recours aux droits}

Social representations and experiences of justice: The example of non-take up of rights

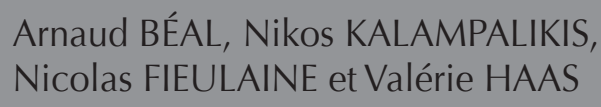

Laboratoire GRePS (EA 4163), Université Lyon 2, France 


\section{Expériences de justice et représentations sociales : l'exemple du non-recours aux droits}

Notre article se focalise sur une problématique émergente en sciences sociales, celle du non-recours au droit. À partir des apports sociologiques et psychosociaux classiques et récents nous proposerons une investigation des rapports des citoyens à la justice et aux droits sociaux sous l'angle de l'approche des représentations sociales. Des données qualitatives et quantitatives recueillies auprès du terrain et de populations vulnérables illustreront les injonctions paradoxales dont les populations fragiles font l'objet, intériorisation d'un statut disqualifiant tout en le revendiquant pour s'en affranchir. À cette fin, nous proposerons d'étudier de l'intérieur de leur lieu de production, circulation et diffusion la logique de ces représentations fragilisées dans leur rapport au droit.

\section{Social representations and experiences of justice: the example of non-take up of rights}

Our paper focuses on an emerging issue in the social sciences: the non-take up of rights. Based on classic and more recent sociological and psychosocial research, we used the social representations approach to explore the citizens'relationships to justice and to their social rights. Qualitative and quantitative field data were collected from vulnerable populations (in the region of Lyon, France). The results highlighted the paradoxical injunctions to which these vulnerable populations were subject, such as having to internalize a disqualifying status while having to claim it in order to overcome it. To explore this further, we suggest to study "from the inside " where and how the logic of such representations vulnerable in their relationship to law were produced, circulated and diffused.

\section{Erfahrungen mit Gerechtigkeit und sozialer Repräsentation : Das Beispiel des Nicht-in- Anspruch-nehmen von Rechten}

Unser Beitrag richtet sein Augenmerk auf eine seit Jahren in den Sozialwissenschaften auftretende Problematik des Nicht-in-Anspruch-nehmen von Rechten. Bezug nehmend auf soziologische (Warin, 2007 ; Odenore, 2012), klassisch psychosoziale (Robert \& Faugeron, 1978 ; Felstiner, Abel \& Sarat, 1981) und neueren Beiträge (Dubouchet, 2004 ; Fieulaine, Kalampalikis, Haas, 2009 ; Fieulaine, Kalampalikis, Haas \& Béal, 2013) schlagen wir eine Erforschung der Reports von Bürgern zur Gerechtigkeit und zu sozialen Rechten unter dem Gesichtpunkt eines Ansatzes der sozialen Repräsentation vor (Moscovici, 2013). Die qualitativen und quantitativen Daten, die in diesem Bereich bei prekären Bevölkerungsschichten gesammelt wurden, zeigen die paradoxen Denkmuster, von denen prekären Schichten Gebrauch machen. Sie verweisen auf eine Verinnerlichung des disqualifizierenden Status, bei dem all das herabsetzt wird, das einzufordern ist, um davon frei zu kommen. Daraus folgernd schlagen wir vor, den inneren Bereich der Entstehung, der Zirkulation und Diffusion, wie auch die Logik dieser fragilen Repräsentation in ihrem Verständnis des Rechts zu untersuchen. 
Experiencias de justicia y representaciones sociales : el ejemplo del no-recurso a los derechos

Nuestro artículo se focaliza en una problemática emergente hace algunos años en ciencias sociales, la del no-recurso al derecho. A partir de aportes sociologicos (Warin, 2007; Odenore, 2012) y psicosociales clásicos (Robert \& Faugeron, 1978 ; Felstiner, Abel \& Sarat, 1981) y recientes (Dubouchet, 2004 ; Fieulaine, Kalampalikis, Haas, 2009 ; Fieulaine, Kalampalikis, Haas \& Béal, 2013) proponemos una investigación sobre las relaciones de los ciudadanos con la justicia y con los derechos sociales según el enfoque de las representaciones sociales (Moscovici, 2013). Los datos cualitativos y cuantitativos recogidos en el trabajo de campo y con poblaciones vulnerables, ilustran las paradojas de las cuales son objeto las poblaciones frágiles, interiorización de un estatus descalificante reivindicado justamente para liberarse. En este sentido, nos propusimos estudiar desde el interior de su lugar de producción, circulación y difusión, la lógica de estas representaciones fragilizadas en su relación al derecho.

\section{Experiências da justiça e representaçoes sociais : o exemplo do nao recurso aos direitos}

O nosso artigo aborda questões emergentes desde há alguns anos nas ciências sociais, do não recurso ao direito. A partir das contribuições sociológicas (Warin, 2007; Odenore, 2012) e psicossociais clássicas (Robert \& Faugeron, 1978; Felstiner, Abel \& Sarat, 1981) e recentes (Dubouchet, 2004; Fieulaine, Kalampalikis, \& Haas, 2009; Fieulaine, Kalampalikis, Haas \& Beal, 2013) propomos uma investigação dos relatórios dos cidadãos à justiça e direitos sociais em termos da abordagem de representações sociais (Moscovici, 2013). Dados qualitativos e quantitativos recolhidos no terreno e em populações vulneráveis ilustram as injunções paradoxais de que são objeto as populações frágeis, interiorizando uma desqualificação do estatuto, requerendo-o para se libertarem. Para tal, propomos a estudar o interior do seu local de produção, circulação e divulgação, a lógica dessas representações fragilizadas na sua relação ao direito.

\section{Esperienze di giustizia e rappresentazioni sociali : I'esempio del non riccorso al diritti}

II nostro articolo si focalizza su una problematica emergente negli ultimi anni nelle scienze sociali, quella del non ricorso alla giustizia. A partire dal contributo di alcuni sociologi (Warin, 2007 ; Odenore, 2012) e dal contributo di ricercatori in psicologia sociale ormai classici (Robert \& Faugeron, 1978 ; Felstiner, Abel \& Sarat, 1981) o più recenti (Dubouchet, 2004 ; Fieulaine, Kalampalikis, Haas, 2009 ; Fieulaine, Kalampalikis, Haas \& Béal, 2013) proponiamo l'idea di esplorare i rapporti tra i cittadini e la giustizia e il diritto sociale dal punto di vista dell'approccio delle rappresentazioni sociali (Moscovici, 2013). Dati sia qualitativi che quantitativi raccolti nell'ambito di popolazioni vulnerabili, mostrano in che modo le popolazioni fragili siano oggetto di ingiunzioni paradossali, nel momento in cui interiorizzano uno status sociale squalificato mentre rivendicano la loro lotta per affrancarsene. Proponiamo dunque di studiare la logica di tali rappresentazioni fragili della propria relazione con il mondo del diritto, ponendoci all'interno del loro stesso luogo di produzione, circolazione e diffusione. 
La correspondance pour cet article doit être adressée à Arnaud Béal, Université Lyon 2, Institut de Psychologie, Laboratoire GRePS (EA 4163), 5 avenue P. Mendés-France, 69656 Bron, France ou par courriel à <arnaud.beal@univ-lyon2.fr>.

Contribution des auteurs :

Arnaud Béal : conception et réalisation des travaux, analyse et interprétation des résultats, rédaction de l'article ;

Nikos Kalampalikis : conception et réalisation des travaux, analyse et interprétation des résultats, rédaction de l'article ;

Nicolas Fieulaine: conception et réalisation des travaux, analyse et interprétation des résultats, rédaction de l'article ;

Valérie Haas : conception et réalisation des travaux, analyse et interprétation des résultats, révision de l'article.

Ce projet a été réalisé grâce une subvention accordée par la Mission de Recherche « Droit \& Justice » (GIP) (2008-10) et une bourse de thèse (CDU) accordée à Arnaud Béal (2010-2013) par la Région Rhône-Alpes. 


\section{Le non-recours : apparition et évolution d'une catégorie d'analyse}

Le " non-recours » est une notion émergente et de plus en plus présente dans le champ politique et médiatique français ces dernières années, contiguë à son développement dans des recherches récentes en sciences sociales (cf. Observatoire des non recours aux droits et services - Odenore, 2012). Issue d'études portant sur l'effectivité des politiques de prestations sociales, la notion de non-recours correspond à l'origine à la situation de toute personne éligible à une prestation sociale, qui - en tout état de cause - ne la perçoit pas. Elle vient désigner des pratiques, ou plutôt l'absence de pratiques, concernant, particulièrement, les dispositifs de protection sociale liés à la " lutte contre la pauvreté et l'exclusion ». L'apparition de cette catégorie d'analyse des pratiques citoyennes face aux droits varie en fonction des histoires nationales et des systèmes de solidarité institutionnelle. Toutefois, partout où elle a émergé, elle est corrélative à l'évolution des systèmes de protection sociale et en particulier des conditions d'éligibilité et d'accès aux prestations vers une moindre générosité et un plus grand contingentement des offres de droits, mais aussi des services qui en assurent l'accès.

Deux principes ont ainsi structuré l'offre de droits ces dernières décennies : un plus grand ciblage de l'offre, dès lors plus sélective et désignant des catégories de bénéficiaires (Warin, 2010a); et l'activation des prestations, c'est à dire leur mise sous conditions de participation active des bénéficiaires, dans la logique du mérite des droits (Vrancken, 2010). Ainsi la problématique du " non-recours » s'ancre dans la transformation des «États-providence » que l'on observe depuis les années 70 dans les pays industriels avancés, dans lesquels les niveaux de couverture des différents risques sociaux ont été réduits ces trente dernières années (Castel, 2009 ; Wacquant, 2007; Warin, 2007). Les droits sociaux universels et assurantiels ont vu leur part se réduire au profit de prestations et de dispositifs destinés à des publics spécifiques. Cette notion est ainsi relativement contemporaine à l'émergence, non moins récente, de l'idée d'exclusion sociale (Castel, 1995). L'accès au(x) droit(s) est devenu un enjeu politique et social, revendiqué par des groupes organisés et relativement pris en compte dans les réformes administratives (Hamel, 2013). ${ }^{1}$

Un article de Catrice-Lorey, datant de 1976, portant sur les effets de la montée en puissance de ces politiques sociales ciblées fait apparaître pour la première fois explicitement en France le terme de non-recours, en tant que facteur à prendre en compte dans la compréhension des inégalités socio-économiques, qui se construiraient aussi via les inégalités d'accès aux systèmes de protection sociale. Mais il faut attendre les années 1990 pour que la thématique du non-recours aux droits sociaux émerge véritablement en France via une approche économétrique en termes de coût/bénéfice (cf. Van Oorschot Math, 1996). Ce cadre d'analyse vise à appréhender le phénomène essentiellement d'un point de vue quantitatif. Il est calculé en faisant la différence entre la population éligible théoriquement et la population bénéficiaire, permettant ainsi d'établir des «taux de non-recours $»^{2}$. 


\section{Extension du domaine du non-recours}

Les travaux menés en France, notamment par l'Odenore ${ }^{3}$, ont permis d'approfondir et d'étendre le domaine d'étude du non-recours, aux droits sociaux universaux et assurantiels, ainsi qu'aux droits et services que l'offre publique rend disponibles (les soins, la justice, la police, etc.). Cette définition élargie implique que les causes du non-recours peuvent se situer dans une absence ou un manque de connaissances, dans un problème de réception de l'offre (quand le droit est connu, demandé, mais non obtenu) ou encore dans une non-demande par désintérêt ou refus, les trois niveaux étant interdépendants (Warin, 2010). Au-delà de la connaissance de I'offre de droit, de son accessibilité, des problèmes relatifs à sa réception, le fait de ne pas demander ses droits alors qu'on les connaît révèle un type de non-recours " inaudible " (Mazet, 2010), teinté de conflictualité. Ce non-recours rend saillant un problème lié à la non-reconnaissance des dispositifs par les sujets de droit. Ainsi, pour " faire valoir ses droits » il faut les connaître, mais il faut également «s'y reconnaître » (Berrat, 2011, p. 139). Selon Warin (op. cit.), le non-recours par non-demande révèle un abandon ou un rejet par les individus de leur " citoyenneté sociale », qui lui-même signale un désaccord ou un désintérêt de ce qui est institué par l'État et/ou les collectivités locales. Il remet en question la pertinence et l'effectivité de ces offres, tout en venant renforcer les inégalités socio-économiques qu'elles sont censées pallier. Le sentiment de stigmatisation induit par le ciblage de l'offre de droits et vécu dans l'action de recourir à certains dispositifs serait un des éléments explicatifs de la non-demande ou de l'abandon du recours. C'est ce que font émerger, notamment, les travaux sur le « welfare stigma » aux États-Unis (cf. Horan \& Austin, 1974 ; Stuber \& Schlesinger, 2006) inscrivant le phénomène du non-recours dans les idées associées à la pauvreté dans la culture américaine, marquée par l'hégémonie de la responsabilité et d'autonomie individuelles (Warin, op. cit., p. 119).

L'acceptation et la reconnaissance de l'identité, du statut et de l'offre de droit sont centrales dans la compréhension des dynamiques de non-recours (Berrat, 2011 ; Mazet, op. cit.). Ainsi, le non-recours aux droits et aux dispositifs liés aux catégories produites dans le cadre de l'action sociale et médico-sociale, constitue toujours une forme de positionnement à leur égard, tant dans leur appropriation que dans leur rejet (Berrat, op. cit., p. 137).

Cet objet d'étude, que nous présentons sommairement dans le cadre de cet article, $\mathrm{s}^{\prime}$ inscrit également et plus largement dans la tradition de recherche en anthropologie et sociologie du droit. Le droit est observé " en acte », à côté du " droit des livres»(Liora, 2009, Pélisse, 2003, 2005 ; Ewick \& Silbey, 1998 ; Sarat, 1990), comme le préconisait déjà la sociologie du droit (Ehrlich, 1936 ; Gurvitch, 1935) qui insistait déjà sur l'importance de l'expérience juridique vécue par les acteurs pour comprendre « la vie du droit ». Dans ce cadre, les conflits autour du droit sont considérés comme des construits sociaux plutôt que des choses (Felstiner, Abel \& Sarat, 1991) : « étudier l'émergence et la transformation des conflits en litiges c'est étudier un processus social tel qu'il survient » (lbid, p.41) et permet d'examiner " des griefs et des conflits qui ne sont jamais institutionnalisés comme litiges (...) 
ceux qui sont encaissés sans rien dire » (Ibid, p.49). Ce courant de la sociologie juridique centré sur l'analyse des processus conduisant au " claim « (cf. Miller \& Sarat, 1981) rejoint sous bien des aspects les recherches plus récentes sur le nonrecours, entre autres à la justice et aux droits (Fieulaine, Kalampalikis, Haas, 2009).

\section{Représentations sociales et non-recours à la justice}

La psychologie sociale, et notamment le courant de recherche s'inscrivant dans la théorie des représentations sociales (Moscovici, 1961, 2013 ; Jodelet, 1989), nous permet de poser un regard original sur le phénomène du non-recours. Notamment car l'étude des représentations sociales se focalise sur les rapports entre savoir expert et savoir profane (Hewstone \& Moscovici, 1984). Elle rend compte de la transformation d'un savoir expert en un savoir ordinaire, chacun trouvant sa pertinence dans des contextes sociaux différents.

Le droit institué socialement est « un autre monde » (Marie-Angèle Hermitte, 2013) et le rapport aux droits constitue un rapport à cet autre monde, à bien des égards étrange à la vie quotidienne, qui a son propre langage, ses règles et ses codes. Les sujets non experts en droit se confrontent à l'altérité quand ils recourent au droit. La dialogicité (Markovà, 2007) entre différentes rationalités du droit, ou pour reprendre la distinction de Max Weber, entre le droit formel et le droit matériel, peut être source de changement autant qu'elle peut produire de la résistance. En cela, étudier le rapport entre la rationalité du droit et celle du sens commun, du point de vue des représentations sociales, peut nous éclairer sur leurs possibilités de communication et sur les possibles effets d'incommunicabilité (Moscovici, 2012) qui peuvent participer aux rejets de la pensée et de la pratique formalisée du droit.

Les travaux sur les rapports entre ces savoirs montrent les ponts et les résistances qui peuvent se construire quand le sens commun doit intégrer les savoirs experts. Nous savons que les connaissances profanes sur la justice institutionnelle sont relativement faibles en quantité (Robert \& Faugeron, 1978, 1980 ; Percheron, 1991 ; Scharnitzky \& Kalampalikis, 2007), fortement stéréotypées et souvent imprégnées par son caractère pénal et criminel (Baraquin, 1979). Pour autant, les manières de penser la justice sont multiples et les sujets novices ne pensent pas la justice selon les critères des professionnels du droit (Clémence \& Doise, 1995, p. 375). Aussi, la justice distributive - perception du caractère juste des récompenses ou rétributions, selon que l'on prend en compte les besoins, le mérite ou l'égalité (Roux \& Clémence, 1999) - et la justice procédurale - en tant qu'évaluation de la justice des procédures de prise de décision qui précèdent et déterminent la distribution des résultats, des ressources, etc. (Azzi, 1998) - ne sont pas pensées avec la même facilité par les sujets non-experts. La justice distributive, inscrite dans des théories naïves de la justice, semble davantage correspondre à la pensée de sens commun, étant caractérisée par le fait que la conclusion prime sur l'analyse, le processus (Hewstone \& Moscovici, op. cit.), comparativement à la justice procédurale qui implique de prendre en compte le processus qui a amené à la distribution. Les procédures relatives à l'application du droit seraient ainsi bien moins connues, rendant saillante la plus grande difficulté pour la pensée de sens commun à s'appro- 
prier le savoir relatif à la justice procédurale. Ce qui pourrait expliquer certaines difficultés à s'approprier le savoir nécessaire concernant les procédures d'accès aux droits, pouvant entrainer du non-recours par incompréhension des procédures par lesquels passer pour recourir, ou si les sujets ne s'en remettent pas aux experts, via les lieux d'accès aux droits.

Mais la difficile ou relative appropriation du savoir expert dans le sens commun n'empêche pas les sujets de penser la justice et le droit. Si les conceptions ordinaires de la justice sont largement imprégnées des textes institutionnels (des Droits de l'Homme notamment), elles sont structurées selon des principes qui reposent surtout sur les normes sociales. D'ailleurs il a été mis en évidence que ces normes sociales agiraient dans les pratiques de justice des experts eux-mêmes (Amsterdam \& Bruner, 2000 ; Doise, 2007 ; Hunout, 1987). Le raisonnement propre aux représentations sociales de la justice permet aux sujets de définir la juste répartition des droits dans des contextes particuliers et concrets, selon les normes d'équité, d'égalité stricte, du besoin. C'est ainsi que les travaux sur les représentations sociales des droits sociaux (Jaquemain, 1995) ou des Droits de l'Homme (Doise, 2001, 2009) montrent que dans des contextes sociaux, leur application est soumise à des logiques psychosociales, marquées notamment par l'égo- ou le socio-centrisme (Clémence \& Doise, 1995).

Les travaux sur les représentations sociales de la justice rejoignent l'interrogation sur les conceptions du rapport entre individus et État et le positionnement visà-vis de l'universalité des droits (Clémence, Doise, de Rosa, Gonzalez, 1995). L'adhésion à la conception du libre marché - norme du mérite individuelle et distinction interindividuelles entre gagnants et perdants - et/ou à celle des inégalités structurelles - distinctions de classes sociales et causes externes aux inégalités sociales - fonctionnent comme des représentations sociales normatives pour déterminer qui a droit à quoi et revendiquer plus ou moins d'intervention de l'État face aux inégalités sociales (État minimal ou redistributeur) (Staerklé, Delay, Gianettoni, Roux, 2007). Le rapport idéel et pratique à la justice et au droit s'inscrit dans la réflexion menée sur la légitimation des inégalités sociale (Jost \& Banaji, 1994). Si les conceptions du vivre-ensemble, dans lesquelles interviennent des attributions causales (internes ou externes) et des distinctions sociales (interindividuelles ou intergroupes), agissent en tant que normes pour se positionner vis-à-vis de l'intervention de l'État, nous pouvons nous questionner sur le lien entre les conceptions des rapports sociaux, les positionnements vis-à-vis de l'intervention de l'État et les pratiques de recours au droit que ces représentations peuvent instituer; lien entre représentations sociales de la justice et son usage de la justice déjà mis en évidence par ailleurs concernant les représentations sociales de l'appareil judiciaire (Fieulaine, Kalampalikis, Haas, Beal, 2012).

Les représentations sociales, en tant que savoirs populaires inscrits dans notre langage, dans lequel la société peut puiser le cas échéant, en fonction des situations, sont dépendantes de l'essentiel de nos pratiques sociales, de nos expériences quotidiennes du monde (Moscovici, 2013). C'est d'ailleurs l'une de leur fonction 
essentielle, d'être avant tout un guide pour comprendre, juger et agir sur et dans le monde. Elles détiennent une certaine autorité, définissant tout ce qui est considéré comme licite/illicite, admissible/inadmissible, tant au niveau de ce qui peut être pensé, dit et fait. Elles permettent donc autant de construire notre expérience du monde que de lui donner un sens familier (Jodelet, 2006). Mais que se passe-t-il quand le rapport à notre environnement devient " étrange et dérangeant », quand il y a rupture de l'expérience ordinaire (Moscovici, op. cit., p. 109)?

Aussi, nous avons souhaité interroger les facteurs psychosociaux participant au déclenchement et à la construction du recours au droit. Nous interrogeons particulièrement la place d'autrui et ainsi le rôle du partage social dans les pratiques de droit. Le déclenchement du recours au droit, en tant qu'action, sera également questionné à travers ses aspects temporels. Nous questionnons enfin la place et le rôle des représentations sociales de la justice et du droit dans ces pratiques de (non-)recours au droit et/ou à la justice. Comment se construisent-elles et s'actualisent-elles dans ces contextes? Quelles sont leurs rôles dans les expériences vécues des sujets? Une attention particulière sera mise sur les phénomènes de fragilisation des représentations sociales de la justice et du droit qui s'observent à travers ces expériences juridiques.

\section{Dispositifs méthodologiques et terrains d'enquête}

Afin de répondre à ces questions, nous avons élaboré une recherche empirique de terrain, basée sur une méthodologie variée, nous permettant d'observer notre objet de différents points de vue. Des méthodes de recueil de données et d'analyses qualitatives et quantitatives ont été construites sur plusieurs terrains d'enquêtes et auprès de différentes populations afin de saisir les dynamiques psychosociales des rapports idéels et pratiques au droit et à la justice.

Une première étape de recherche visait à explorer, dans une optique psychosociale, les cheminements qui conduisent du conflit au (non)recours à la Justice, en réactualisant des perspectives de recherches proposées il y a une trentaine d'années et quelques peu délaissées depuis (Dubouchet, 2004). Le déroulement de cette étape $\mathrm{s}^{\prime}$ est organisé autour de trois phases, quantitatives et qualitatives. La première a constitué en l'exploration des représentations sociales de la justice dans un échantillon de jeunes étudiants français en sciences humaines et sociales $(n=263)$, à partir d'une réactualisation du questionnaire construit à la fin des années 70 par Robert et Faugeron (1978). Simultanément, nous avons investit pendant un temps long des terrains de l'accès aux droits de l'agglomération lyonnaise : les Boutiques de Droit (BD) et les Maisons de Justice et du Droit (MJD). La justice a pu y être observée comme objet d'expérience. Le dispositif méthodologique se centrait sur les justiciables et les contextes de recours, dans ces lieux susceptibles de nous permettre d'accéder à des formes de non-recours. Ces structures, de part leur gratuité, se tournent essentiellement vers des personnes en situation de précarité, mais sont ouvertes à tous. Elles se présentent comme des terrains où les justiciables mobilisent des tiers (des juristes porteurs de ressources sociales et de savoirs experts) afin de rechercher les voies d'une résolution du (ou des) conflit(s) dont ils font l'expé- 
rience. Mais ce sont des terrains infrajudiciaires se situant en amont du recours à la justice ou parfois comme des terrains parajudiciaires, situés en parallèle. Ainsi, le non-recours à la justice que ces terrains offrent potentiellement à voir peut être distingué du recours aux droits qui peut exister néanmoins distinctement. Un dispositif d'observations des consultations d'accès aux droits $(n=17)$, ont été complété par une série d'entretiens menés avec les acteurs des lieux d'accès aux droits $(n=9)$ et d'une série d'entretiens avec les usagers des lieux $(n=47)$ et enfin d'un questionnaire ciblés auprès des usagers de ces mêmes lieux ( $n=132$, mage $=43,3, \sigma=14,5$; $57 \%$ hommes, $43 \%$ femmes). Ce premier dispositif méthodologique nous a permis de cartographier le sens donné à la démarche de l'accès au droit, ses cadres temporels, ses logiques d'action et de faire-face (coping), son vocabulaire, ses éventuelles stratégies de recours et de résolutions.

Dans un deuxième temps, une enquête de terrain sur ces mêmes lieux a été construite en se focalisant cette fois sur des problématiques spécifiques de justice sociale, liées à des difficultés matérielles que rencontrent les sujets et pour lesquelles ils viennent chercher une solution. Les recours à ces structures d'accès au droit sur lesquels nous nous sommes focalisés étaient spécifiquement liés à l'émergence de problèmes matériels, impliquant la revendication ou la défense de certains droits sociaux ou d'aides sociales. Ainsi, trois BD et une MJD de l'agglomération lyonnaise ont été ciblés si situant dans des territoires où les usagers occupent statistiquement davantage des situations basses dans la hiérarchie sociale (employés, ouvriers, sans emplois) et présentent statistiquement plus de problèmes relatifs à la réception des droits sociaux (droits du travail, problèmes avec les administrations, etc.) ${ }^{4}$. Parallèlement aux observations des consultations d'accès aux droits effectuées pendant quatre mois, une nouvelle série d'entretiens a été effectuée auprès d'usagers $(n=16)$.

Enfin, un dernier temps d'enquête de terrain à été effectuée au sein d'un autre type d'accès gratuit aux droits, à destination de personnes en situation de pauvreté et ayant la spécificité de ne pas avoir d'adresse fixe : I'Association Lyonnaise d'Ingénierie Sociale (ALIS). Elle offre la possibilité à ses usagers d'avoir une adresse, de recevoir du courrier, et ainsi de commencer ou de continuer à faire des démarches de droits. Des travailleurs sociaux y assurent l'accès au droit et apportent leur aide à la résolution des problèmes rencontrés par les usagers. Cette structure permettait d'effectuer une observation auprès d'une population relativement "sensible », permettant d'observer les dynamiques d'exclusion dans les rapports aux droits. La population « sans domicile fixe » que cette association accueil doit être comprise dans toute sa complexité et sa diversité. 15 entretiens semi-directifs y ont été effectués, ainsi qu'une " observation multiple» (Chauchat, 1995, p. 95) pendant trois mois et demi.

L'ensemble de ces temps d'enquête et l'analyse des données quantitatives et qualitatives nous permettent de faire émerger une diversité de facteurs psychosociaux explicatifs des dynamiques de recours aux droits et à la justice, notamment marquée par le rôle des représentations sociales. Nous présentons ci-après quelquesunes de ces dimensions les plus saillantes, avant de les discuter. 


\section{Le partage social et le recours au droit}

Les différentes étapes de recherche mettent en évidence, de manière saillante, l'importance d'autrui dans les dynamiques de recours aux droits. Le partage social, ou le fait de communiquer avec autrui sur sa situation, apparait être un facteur décisif dans le déclenchement du recours. Cela est particulièrement saillant dans les résultats transversaux issus aussi bien des entretiens avec les acteurs qu'avec les publics des BD/MJD. Le rôle de l'entourage a été formellement mis en évidence via le questionnaire diffusé auprès de 132 usagers de ces structures qui proposait une série d'items relatifs aux motifs du déclenchement du recours, présentés dans le tableau 1 (ci-dessous). S'il n'est pas nécessairement l'initiateur - 26\% disent seulement avoir été poussé par leur entourage pour recourir -, il est davantage le moteur du recours. Afin d'étudier comment s'organisent les différents motifs proposés, nous avons réalisé une analyse en composante principale (ACP avec rotation varimax) sur les 15 items portant sur les motifs de recours. La solution factorielle fait apparaître deux facteurs (scree test, Cattell, 1966) expliquant au total 43,7 \% de la variance. Le premier facteur rassemble les items liés aux marqueurs temporels et à la prise de conscience du problème rencontré, tandis que le second se centre sur

Tableau 1 : items et facteurs du déclenchement du recours

\begin{tabular}{lccccc}
\hline Items & F1 & F2 & M & SD & D'accord \\
\hline 6. Je me suis dit que ça n'allait jamais s'arrêter &, 76 & 3,16 & 1,65 & $51 \%$ \\
7. Ça durait depuis trop longtemps &, 76 & 3,23 & 1,66 & $54,2 \%$ \\
12. J'ai pensé à ce que allait être l'avenir si je ne faisais rien &, 66 & 3,73 & 1,52 & $65,4 \%$ \\
8. J'ai décidé de changer la situation &, 66 & 3,98 & 1,47 & $73,2 \%$ \\
4. Ce que je faisais jusque-là n'a servi à rien &, 63 & 3,20 & 1,66 & $48,5 \%$ \\
11. J'ai regretté de n'avoir rien fait jusqu'ici &, 62 & & 3,18 & 1,66 & $47,2 \%$ \\
9. J'ai peur que ma santé soit atteinte par ce problème &, 61 & & 2,79 & 1,72 & $39,3 \%$ \\
2. La situation est devenue insupportable &, 57 &, 37 & 3,62 & 1,59 & $58,5 \%$ \\
14. Je me suis rendu compte que ma situation était injuste &, 55 &, 35 & 3,85 & 1,57 & $68,1 \%$ \\
5. J'ai su qui était responsable de ma situation &, 53 &, 36 & 3,33 & 1,65 & $54,3 \%$ \\
10. J'ignorais que je pouvais faire quelque chose &, 44 & & 2,50 & 1,59 & $30,5 \%$ \\
15. La situation a changé brusquement &, 34 & & 2,71 & 1,65 & $34,7 \%$ \\
3. J'ai discuté avec mon entourage de mon problème & &, 83 & 3,38 & 1,60 & $49,5 \%$ \\
1. Mon entourage m'a poussé à le faire & &, 76 & 2,31 & 1,66 & $26 \%$ \\
13. Je me suis aperçu que d'autres gens avaient le même & &, 49 & 2,98 & 1,65 & $45,9 \%$ \\
problème & 5,25 & 1,30 & & & \\
\hline Valeur propre & 34,98 & 8,70 & & & \\
\hline Variance expliquée & & & & & \\
\hline
\end{tabular}


les aspects liés au partage social. II apparaît plus significatif encore que les items qui saturent sur les deux facteurs (saturations complexes) correspondent à différentes étapes du processus menant au « claiming » telles que décrites par Felstiner et al. (1981), à savoir la prise de conscience d'une injustice vécue (le naming), et la désignation d'un responsable (le blaming).

Ainsi, discuter avec son entourage du problème dont on fait l'expérience apparaît être une dimension importante dans la formalisation du conflit, dans le sentiment de partager ce problème avec d'autres et dans la désignation d'un responsable. Les interactions au sujet du problème rencontré peuvent agir dans la prise de conscience de son caractère conflictuel, le rendant davantage insupportable, tout en donnant des moyens de mieux maitriser la situation, en l'expliquant et en la relativisant. La relativisation que permet le partage de son expérience, rend aussi envisageable sa réinscription dans des enjeux sociaux, permettant de se rendre compte que sa "situation était injuste ». Les autres participent donc à l'émergence et à la transformation des conflits - en aidant à nommer l'injustice et à désigner un responsable - et donc au processus de recours aux droits. Ils semblent être une des voies d'apaisement de la tension crée par l'expérience d'un problème, le sentiment d'injustice et la rupture du quotidien. Les entretiens effectués avec des usagers de $\mathrm{BD} / \mathrm{MJD}$ illustrent ce processus, comme l'exprime par exemple Josiane ${ }^{5}$ :

" - J'ai des amis, ils m'ont dit: «Josiane, il faut te plaindre. T'es en droit.» Donc on m'a poussé, on m'a poussé et c'est pour ça que je me plains. D'accord. - Parce qu'on m'a dit que j'étais en droit, vu mon handicap, vu ce que j'ai eu, que on, ils pouvaient pas m'envoyer travailler. Que je pouvais pas. De toutes façons je pouvais pas - D'accord. - Voilà. Donc c'est avec l'aide de mes proches qui me soutiennent, qu'on ouvre les yeux quoi. »

Néanmoins, les recours aux droits sociaux observés dans l'association ALIS montrent que le partage social à son entourage proche de sa situation et des problèmes matériels vécus est souvent rendu difficile du fait de la stigmatisation sociale que certaines situations comportent. Les sujets partagent alors plus facilement leurs difficultés et leurs besoins avec des personnes vivant une expériences analogues et/ou avec des professionnels de l'aide.

C'est également souvent dans l'objectif de partager socialement leur expérience et ce qui fait problème dans leur quotidien que les usagers des BD/MJD ou d'ALIS s'adressent aux lieux d'accès aux droits et à leurs acteurs. L'idée de ne plus être seul face à son problème, le besoin d'écoute, la recherche d'un interlocuteur, sont souvent évoqués par les usagers interrogés venant consulter les professionnels du droit. Le sentiment de reconnaissance que ces derniers offrent aux usagers est régulièrement décrit, représentés comme un entourage familier et permettant de faire face à sa situation.

Plus pragmatiquement, à travers le rapports aux professionnels du droit, les sujets expriment le besoin de trouver une issue institutionnelle, formelle à leur problème, dans sa possible appropriation par la logique du droit et le discours porté par les 
professionnels. Il y a la recherche d'un ancrage dans le discours expert, mais qui n'apparait pas toujours possible, de part son cadre rigide, sa temporalité, ses catégories d'action. C'est la tension qu'exprime Akio ${ }^{6}$ dans l'extrait d'entretien ci-dessous :

«- Donc je répète, je suis venu ici donc, un, pour valider ce que j'ai déjà comme conclusion. Est-ce qu'on a le même point de vue sur les conclusions que j'ai constaté. - Vous veniez ici pour valider un peu. - J'ai déjà une idée a priori de tout ce qui va se passer. Mais en même temps aussi peutêtre est-ce que je me trompe ou je me trompe pas. »

\section{Perspectives temporelles et recours au droit}

Nos résultats mettent également en évidence le rôle capital joué par la perspective temporelle dans la construction du (non-)recours au droit, comme l'indique le premier facteur identifié concernant les marqueurs temporels du recours. $Y$ apparaissent la durée rétrospective du conflit - « ça durait depuis trop longtemps » - des regrets vers le passé - de n'avoir rien fait - et des craintes vers le futur, produite par le fait de se projeter dans une inaction. Inscrivant les recours dans une logique temporelle qu'on peut assimiler à un regret anticipé (Abraham \& Sheeran, 2003) - si je ne fais rien, je vais le regretter - les perspectives temporelles semblent donc jouer un rôle saillant dans le déclenchement du recours au droit via les BD/MJD afin de changer sa situation. Ce facteur est associé aux trois items relatifs à deux des trois étapes du conflit : le naming et le blaming (Felstiner et al., op. cit.), qu'il partage avec le second facteur.

Aussi nous avons pu observer que les perspectives temporelles jouent un rôle dans l'orientation, et donc le sens, du recours. S'il y a recours, celui-ci répond à des motifs différents selon l'orientation temporelle à laquelle il correspond. Orienté vers le passé (la réparation), le présent (I'urgence immédiate) ou le futur (I'anticipation des difficultés à venir), et sera dès lors plus ou moins en contradiction avec la temporalité des dispositifs de droit.

Le recours aux droits pour la résolution d'un conflit et/ou pour faire face à des difficultés implique davantage une projection dans l'avenir. Cela est particulièrement vrai concernant le recours à l'offre publique de droits sociaux et d'aides sociales, de manière générale. L'omniprésence de la notion de projet qui conditionne dans de nombreux dispositifs les droits sociaux assistantiels, peut produire un désaccord temporel entre les bénéficiaires et l'offre (orientée elle vers le futur de manière normative et axiologique).

Cela est particulièrement saillant dans les données recueillies auprès des usagers d'ALIS. Le sentiment de vulnérabilité individuelle - craindre que sa situation se dégrade - que ressentent un certain nombre d'entre eux, construit une activation et une responsabilisation individuelle vis-à-vis de leur situation. Perçue comme dangereuse - si elle s'aggrave ou si elle perdure - elle produit chez ces individus une projection dans l'avenir qui va de pair avec l'idée de "s'accrocher », de " résister » pour « s'en sortir ». L'idée d'avoir la « force », le « mental », d'être « résistant » et 
dans une pratique de protection de sa santé, participe à cette projection dans le futur participant aux droits afin de faire face à la menace de «tomber plus bas ». Nous voyons se profiler ici un lien relativement étroit entre la perspective temporelle future, le sentiment de contrôle de sa situation et la crainte du stigmate qui s'active simultanément à travers l'image d'une sous-catégorie de pauvres associée à la « faiblesse », à la figure du " perdant » de la société libérale.

\section{Représentations de la justice et recours aux droits}

Les recours aux lieux d'accès aux droits, s'ils sont animés par un besoin de régler un problème individuel, s'inscrit dans des représentations sociales de la justice. Le recours aux BD/MJD s'ancre dans des représentations sociales du système judiciaire. Une partie du questionnaire proposé aux usagers des BD/MJD intégrait un ensemble d'items, issus d'un questionnaire explorant les représentations sociales de la justice (Robert et Faugeron, 1978, Fieulaine et al., 2013). Dans cet échantillon d'usagers des BD/MJD, les fonctions de la justice, ainsi que ses défauts de fonctionnement (longueur, complications, coût) sont les éléments les plus approuvés (pourcentage d'accord). Au contraire, les propositions les plus idéalisatrices et défiantes sont celles sur lesquels les sujets s'accordent le moins (cf. Tableau 2 ci-dessous).

Une analyse factorielle réalisée sur les réponses au questionnaire explorant les représentations sociales de la justice (ACP avec rotation varimax) met en évidence quatre facteurs distincts (Scree test ; 43,88 \% de variance expliquée. Un premier facteur est relatif à la défiance à l'égard de l'appareil judiciaire, désignant des pratiques institutionnelles arbitraires et une certaine crainte à son égard - p.e. "les décisions de justice sont presque toujours défavorables aux plus démunis ». Un second facteur est marqué, au contraire, par son idéalisation, lié à une image positive et juste de cette institution - p.e. " De nos jours la justice est proche des citoyens ». Le troisième facteur fait apparaitre un ensemble de propositions lié à la fonction régulatrice et punitive de la justice (rappeler les règles, punir, réguler), ainsi que les défauts de sa réalisation (longueur, complexité) - p.e. "Le fonctionnement de la justice est trop compliqué ». Ces trois premiers facteurs rejoignent ceux identifiés lors d'une recherche précédente auprès d'étudiants en sciences humaines, confirmant l'intérêt de l'outil de mesure utilisé. Malgré tout, un dernier facteur vient s'ajouter dans cet échantillon spécifique, qui met en cause de manière spécifique l'accessibilité de la justice", pouvant conduire à l'évitement - p.e. " c'est trop impressionnant de s'adresser à la justice ». Les entretiens semi-directifs menés auprès des usagers des BD/MJD et d'ALIS, confirment ces résultats concernant les représentations de l'appareil judiciaire. Nous voyons ainsi clairement émerger des indicateurs spécifiques de potentiels non-recours à l'institution judiciaires, à cause de la crainte qu'elle évoque ou relative à son manque d'accessibilité.

Au-delà de ces représentations sociales de l'appareil judiciaire, largement marquées par son caractère pénal, ce sont plus largement des représentations sociales du vivre-ensemble et de l'Etat qui émergent dans les entretiens effectués dans la deuxième phase d'enquête, avec des usagers des BD/MJD et d'ALIS, rencontrant des problèmes socio-économiques et recourant au système de solidarité sociale. 
Tableau 2 : items relatifs aux représentations du système judiciaire

\begin{tabular}{|c|c|c|c|c|c|c|c|}
\hline Items & F1 & F2 & F3 & $\mathrm{F} 4$ & M & SD & D'accord \\
\hline $\begin{array}{l}\text { 4. Les décisions de justice sont presque toujours défavorables } \\
\text { aux plus démunis }\end{array}$ & ,69 & & & & 3,27 & 1,27 & $40 \%$ \\
\hline $\begin{array}{l}\text { 13. La surcharge des tribunaux amène la Justice à prendre de } \\
\text { mauvaises décisions }\end{array}$ & ,69 & & & & 3,50 & 1,28 & $50 \%$ \\
\hline 14. Quand on est face à la Justice, on n'a plus aucun droit & ,69 & & & & 2,45 & 1,36 & $21,4 \%$ \\
\hline 22. Je me méfie de la justice &, 69 &,- 31 & & & 2,82 & 1,37 & $27,6 \%$ \\
\hline $\begin{array}{l}\text { 25. Dans une affaire, dès les premiers contacts avec la justice, } \\
\text { les jeux sont faits }\end{array}$ & ,66 & & & & 2,71 & 1,30 & $21,4 \%$ \\
\hline 20. Dès que l'on a à faire à la Justice, on se sent soupçonné &, 64 & & & & 3,16 & 1,32 & $39 \%$ \\
\hline 9. Il faut éviter d'avoir affaire à la justice, même si on est innocent & ,60 & & & & 3,06 & 1,69 & $45,1 \%$ \\
\hline 5. De nos jours la justice est proche des citoyens & & ,74 & & & 3,14 & 1,21 & $33 \%$ \\
\hline 2. Si l'on est innocent on a toujours un recours & & ,71 & & & 3,70 & 1,26 & $58,6 \%$ \\
\hline $\begin{array}{l}\text { 19. La justice s'est beaucoup rapprochée des citoyens ces } \\
\text { dernières années }\end{array}$ & & ,67 & & & 3,14 & 1,14 & $30,3 \%$ \\
\hline 10. Les citoyens sont égaux devant la justice & & ,63 & & & 2,99 & 1,48 & $34,7 \%$ \\
\hline 1. La justice permet de faire respecter les droits de l'homme & &, 59 & & & 4,02 & 1,26 & $68,5 \%$ \\
\hline 24. La Justice sert d'abord aux victimes à obtenir réparation & &, 51 & & & 3,62 & 1,25 & $50 \%$ \\
\hline 15. Le langage de la justice est clair & & ,49 & &,- 48 & 2,33 & 1,32 & $16,5 \%$ \\
\hline 6. Le fonctionnement de la justice est trop compliqué & & &, 65 & & 4,29 & 91 & $80,7 \%$ \\
\hline 23. Les procédures judiciaires sont trop longues & & &, 60 & & 4,43 & 89 & $81,7 \%$ \\
\hline $\begin{array}{l}\text { 11. Le rôle de la justice est de faire respecter les droits des } \\
\text { citoyens }\end{array}$ & &, 31 &, 53 & & 4,48 & ,98 & $85,1 \%$ \\
\hline $\begin{array}{l}\text { 16. Le rôle de la justice est de rappeler que les règles de la } \\
\text { société doivent être respectées }\end{array}$ & & &, 52 & & 4,18 & 1,12 & $74,5 \%$ \\
\hline 26. La justice n'est pas assez sévère avec ceux qui entravent la loi & & &, 51 & & 3,50 & 1,19 & $44,3 \%$ \\
\hline $\begin{array}{l}\text { 21. S'il n'y avait pas la justice, n'importe qui pourrait faire } \\
\text { n'importe quoi }\end{array}$ & & & ,39 & & 4,60 &, 77 & $87,8 \%$ \\
\hline 12. C'est trop impressionnant de s'adresser à la justice & & & & ,68 & 3,56 & 1,42 & $56 \%$ \\
\hline $\begin{array}{l}\text { 8. J'hésiterais à engager une action en justice si j'étais victime } \\
\text { d'une infraction }\end{array}$ & & & &, 53 & 2,95 & 1,40 & $36 \%$ \\
\hline 18. J'aurais peur de la justice si je devais avoir à faire à elle &, 47 & & & 49 & 3,13 & 1,34 & $39,2 \%$ \\
\hline 17. Pour la plupart des gens, la justice est inaccessible & & & &, 42 & 3,57 & 1,19 & $53 \%$ \\
\hline 3. Ça coûte cher de s'adresser à la justice & & & &, 34 & 4,06 & 1,10 & $69 \%$ \\
\hline $\begin{array}{l}\text { 7. N'importe quel citoyen peut s'adresser à la cours européenne } \\
\text { de justice }\end{array}$ & & & & & 3,38 & 1,33 & $44,3 \%$ \\
\hline Valeur propre & 5,01 & 3,05 & 1,95 & 1,38 & & & \\
\hline Variance expliquée & 19,28 & 11,76 & 7,51 & 5,32 & & & \\
\hline
\end{tabular}


L'idéalisation de l'intervention de l'Etat pour réguler les rapports sociaux s'oppose à des formes de critiques inscrites dans des théories naïves de la justice distributive et procédurale. Si les procédures d'accès au droit peuvent être perçues comme justes par certains sujets, car compréhensible, simple, elles peuvent, a contrario, être difficilement objectivées, et ancrées à l'envers, décrites à travers les stigmates de la maladies, de la folie, de l'inhumanité. Cette représentation de l'accès aux droits inscrit le recours au droit dans un rapport de pouvoir, allant jusqu'à la construction d'une théorie du complot contre les ayants droit, créant de fortes tension dans les pratiques de droit et un potentiel de non-recours, comme l'évoque Ben ${ }^{8}$ décrivant un « système qui est fait pour que t'en ais marre, avant de t'en sortir ».

Aussi, une représentation d'une société française juste et la croyance en ses institutions régulatrices apparaissent dans certains discours tenus essentiellement par des usagers d'ALIS, s'opposant à une représentation d'une société française injuste (voire du monde) et à une méfiance des institutions régulatrices des rapports sociaux. Kamel ${ }^{9}$, par exemple, estime que les " lois sont bien faites " en France, contrairement à d'autres pays, et que «si on veut s'en sortir on peut ». Cette conception de l'Etat est bâtie sur une négation des inégalités sociales et la croyance en un monde juste (Lerner, 1980). Cependant, cette conception idéalisée de l'Etat français peut être fragilisée quand celui-ci est perçu comme injuste car non équitable, trop égalitaire, ne récompensant pas les bons pauvres, les " méritants » ${ }^{10}$. La théorie de l'abus des droits et des aides sociales est saillante dans cette représentation, dans laquelle la catégorie des étrangers est souvent mêlée à celle des mauvais pauvres, liée à une conception libérale des droits de l'homme et à une remise en cause de leur universalité. J.-P. ${ }^{11}$ exprime ce point de vue :

« Y a des valeurs républicaines à respecter et ça, je n'en démordrai pas. - Qui sont lesquelles ? - Ah oui, (...) c'est sûr, y a la liberté, ça, c'est sûr, égalité : alors égalité, attention hein, fraternité aussi hein, on peut pas mettre, on peut pas mettre tout le monde sur un même pied, ça, c'est pas possible, comme dit Guéant ce matin. »

L'adhésion à cette conception libérale de l'ordre social produit de la tension et un sentiment de stigmatisation dans le recours à certains droits sociaux et ainsi une non-demande axiologique, potentiel ou effectif. J.-P. continue en expliquant sa gêne de recourir à un minima social et évoquant par ailleurs sa non-demande de droits sociaux connexes :

"C'est pas parce que je suis au RSA que je dois vivre, comment je vais dire ça ? Aux crochets d'une. Ça me dérange déjà fortement d'être au RSA. »

Simultanément ou exclusivement, la société et les institutions régulatrices des rapports sociaux peuvent être représentées comme injustes car structurellement inégalitaires. Cette conception de l'ordre social, attribuant les inégalités socioéconomiques au fonctionnement même du système, construit des comparaisons intergroupes entre riches et pauvres, " grands » et " petits », dirigeants politiques et peuple. C'est une véritable dénonciation des inégalités structurelles qui s’oppose 
ou relativise l'injonction à la personnification de la responsabilité de sa situation. Les droits de l'homme ou les valeurs fondamentales sont utilisés avec force afin de dénoncer la différence entre les valeurs universelles de l'Etat et ses pratiques qui $n^{\prime} y$ correspondent pas. De nouveau, le monde vécu devient étrange en rapport avec l'idéal de justice, comme l'exprime encore Ben :

« (...) quand tu vois que le monde il est vraiment comme ça, et ben c'est pas ce que j'appelle la justice quoi. Moi la démocratie, j'appelle pas ça la démocratie. Liberté, égalité, fraternité c'est des beaux mots, mais quand tu vois ce qu'ils font ça devient des gros mots. »

Si l'idée du mérite peut néanmoins être utilisée pour justifier le recours à certains droits, les inégalités socio-économiques sont délégitimées à travers l'idée d'égalité. La justice ou le droit tels qu'ils sont pratiqués ne représenteraient « rien » pour un certain nombre d'interviewés, sans lien avec l'idéal de justice. Ici, les recours aux droits sociaux et aux aides sociales, sont moins ressentis comme illégitimes, les sujets ne s'estimant pas responsables (ou pas seulement) de leur situation. Certains recours aux droits sociaux peuvent alors être décrits comme une revendication politique en tant que dû, voire comme utilitaristes et instrumentaux, marqués par une certaine pratique critique du droit, inscrit dans des rapports de domination. Aussi les sujets n'ont pas ou moins le sentiment d'abuser d'un système structurellement injuste produisant des inégalités et des «faux » ou des « petits » droits. Rosa ${ }^{12}$ explique ce type de recours comme cela :

«Ouais, c'est comme si t'avais pas vraiment de droits et on t'en accorde des petits comme ça qui correspondent pas, enfin qui compensent pas vraiment tous les, tous les empêchements. »

\section{Discussion}

Cet article avait pour objectif d'introduire l'objet d'étude du non-recours dans le champ de la psychologie sociale, nous inscrivant dans une démarche et une réflexion menée par ailleurs dans le champ des sciences humaines et sociales. Nous avons optées pour une démarche ouverte, du point de vue méthodologique et théorique, alimentant notre regard psychosocial des acquis de la sociologie et de l'anthropologie du droit. Pour ce faire, nous avons élaboré un dispositif méthodologique complet sur des terrains relativement sensibles - où les sujets viennent exposer des besoins et tenter de résoudre des problèmes, rétablir un sentiment de justice - afin d'explorer les facteurs psychosociaux intervenant dans le processus de recours au droit, débouchant potentiellement sur des formes de non-recours. La complexité et la richesse des parcours de droit engagent, à partir des cas singuliers, des dynamiques psychosociales contextualisées par les présences actives d'autrui, du passé et du futur, et de représentations sociales partagées.

La temporalité et le partage social de l'expérience vécue sont apparus comme des éléments centraux dans la compréhension du recours au droit. Le processus menant au recours ne peut tout d'abord être envisagé dans un vide social, hors de 
la présence d'autrui. Cette observation centrale revoie à l'idée défendue par Paul Ricoeur qui affirme que « le juste est cet aspect du bon relatif à autrui » (2005, p. 12). Le partage social est décisif dans la formalisation du conflit et dans la possibilité et l'orientation de l'engagement dans un recours aux droits, à la justice ou tout simplement à l'autre. Partager ce qui fait problème avec son entourage et/ou I'exposer à des professionnels permet au problème d'être inscrit, à travers le langage, dans les représentations sociales et/ou dans le savoir expert. Cela implique de le mettre en mots, de le nommer. Le " naming " constitue la première des trois étapes de l'émergence et de la transformation des conflits (Felstiner, Abel, Sarat, 1981 ; Coates \& Penrod, 1981) et l'une des dimensions principales du processus représentationnel (Kalampalikis, 2007). Il permet de donner une existence sociale au sentiment d'injustice, au problème, au besoin vécu subjectivement. Attribuer et partager socialement des significations concernant un objet social donné (réel ou idéel) nécessite d'abord de le dénommer (Kalampalikis, 2002, p. 27). Le partage social de l'expérience subjective rend possible sa socialisation, permet de sortir de la centration sur la personne, dont on sait qu'elle rend plus difficile l'octroie de droits (Clémence et al., 1994 ; Clémence \& Doise, 1995 ; Boltanski, 1990). Il permet de monter en généralité et d'éviter le risque que le sentiment d'injustice soit renvoyé à une vue de l'esprit, voire un syndrome psychiatrique (Boltanski, op. cit.). Le partage social d'une expérience vécue comme problématique doit donc rendre possible le dépassement de ce premier obstacle qui est celui de correspondre aux règles de la normalité (Boltanski, op. cit., p. 23-24). Affronter ce potentiel rejet et cette position d'étrangéité apparaît donc comme le premier obstacle à dépasser pour recourir au droit. Le processus de recours au droit, notamment dans ses formes conflictuelles, se montre alors comme expérience de socialisation (Simmel, 1995).

Si la familiarisation de son problème à travers les représentations sociales est nécessaire pour faire évoluer le conflit et le recours aux droits, celui qui s'effectue ou tente d'être effectué dans le savoir juridique via les professionnels du droit l'est tout autant, voire d'autant plus. La désignation d'une situation comme problématique, injuste, se manifeste également dans le besoin pour les sujets de lui trouver une issue institutionnelle, dans sa possible appropriation par la logique du droit apparaissant comme un " autre monde » (Hermitte, op. cit.). Mais le partage social est rendu relativement plus difficile avec ces derniers qui, malgré leur empathie, utilisent la logique juridique, réifiant le sentiment d'injustice. Ainsi, l'inscription d'un problème vécu dans cette dernière n'est pas toujours envisageable, du fait de son caractère contraignant et formel, de sa temporalité et des catégories de pensée et d'action qu'elle offre. Ce qui montre les possibles incompatibilités entre des revendications de droits et de justice qui peuvent émerger dans la vie quotidienne et ce qui peut être capté et intégré par et dans la logique du droit. Cependant, cette incommunicabilité potentielle n'empêche pas les sentiments d'injustice, les problèmes, les besoins, de continuer d'exister, constituant autant de déplacements vis-à-vis des catégories du droit (Boltanski \& Chiapello, 1999) synonyme de tension et de possible changements sociaux. 
Le partage social doit encore nous interroger sur les difficultés qu'il contient quand il s'agit de partager et de faire reconnaître socialement et légalement un problème potentiellement stigmatisant. Nous avons pu ainsi observer des injonctions paradoxales qui peuvent se produire dans le recours entre la nécessité de s'identifier à un statut dévalorisé, de le revendiquer pour simultanément s'en affranchir (Moscovici, 1996), créant une tension certaine chez les individus et dans leurs relations aux autres. Cela est particulièrement apparu quand les sujets interviewés évoquaient le fait de devoir se plaindre pour obtenir des droits, notamment des droits assistantiels et des aides sociales, associés à la pauvreté, l'exclusion, au handicap, etc. L'exposition publique de sa situation et de ses besoins, peut engager les sujets dans le rejet d'une attitude plaintive, d'une identité en " creux ». Cela renvoie aux travaux sur le welfare stigma évoqué en introduction, sur les frontières mentales et politiques que le ciblage des politiques publiques active, et leur impact sur un nonrecours comme stratégie identitaire (Warin, 2010a). Le partage social nécessitant de mettre en avant l'anormalité d'une situation, tout en recherchant une certaine reconnaissance, révèle une difficulté que certains sujets peuvent ne pas vouloir affronter et se traduisant en non-recours à certains dispositifs..$^{13}$ Ces injonctions paradoxales, qu'impliquent les situations de difficultés socio-économiques, semblent agir plus largement sur les processus de désaffiliation (Castel, 2003, 2009), dans la préférence exprimée par certains sujets de ne pas partager avec leur entourage (amical, familial) les problèmes qu'ils rencontrent.

Le recours au droit et à la justice est aussi caractérisé par son inscription dans le temps. Tenant compte du fait que toute situation vécue ainsi que les intentions qui l'accompagnent s'inscrivent dans une temporalité étendue contenant à la fois le passé (la trajectoire, les souvenirs, la mémoire...), le présent (les situations, les contextes...) et le futur (les projets, les intentions, les aspirations...), les temporalités des recours sont apparues comme des dimensions transversales à prendre en compte. Quel que soit le moment où l'on étudie le recours au droit ou à la justice, il ne s'agit que d'un moment d'une trajectoire de socialisation juridique (KourilskyAugeven, 1990) évoluant au fil des expériences. Les expériences passées de droit et de justice jouent un rôle fondamental dans le rapport entretenu au droit dans le présent. Ainsi, certains sujets à travers leur longue expérience passée de droit, revendiquent un relatif degré d'expertise et d'autonomie dans les démarches de droit, rendant saillant le « savoir expérientiel » (Jodelet, 2013) dans les pratiques de recours ${ }^{14}$. Par ailleurs, les démarches de recours sont à appréhender au regard des perspectives temporelles (Lewin, 1942) dans lesquelles elles s'inscrivent et prennent sens, fondées sur des intentions tournées vers le passé (regret, nostalgie, etc.), le présent (I'urgence, l'immédiat) et/ou vers le futur (changement, résolution, etc.). Nos résultats montrent que la logique temporelle du regret et la direction rétroactive ou proactive qui orientent l'action sont déterminantes dans les attentes à l'égard de l'intervention du droit et/ou de la justice, et ne sont pas sans rappeler les "philosophies de la justice» (cf. Languin, Widmer \& Kellerhals, 2004). Aussi, il est à noter que les temporalités différentes du droit et de la vie quotidienne peuvent être source de non-recours, quand cette dernière ne peut pas s'intégrer dans la 
temporalité du droit. Les décalages qui peuvent se produire entre temps normatif et temps de l'expérience en situation de conflit ou d'insécurité sont sources de difficultés à s'engager dans des démarches de droit. Par ailleurs, dans des situations marquées par la précarité, une forte tension s'exerce entre le temps de l'urgence et le temps du projet (Fieulaine, 2007 ; Fieulaine \& Apostolidis, 2014), produisant un rapport dialogique autant antinomique et paralysant, que dynamique et producteur de changement.

Enfin, un phénomène de fragilisation des représentations sociales de la justice et du droit est mis en évidence dans ses liens avec les pratiques de droit. Cela avait déjà été mis en exergue dès 1978 par Robert et Faugeron, mais aussi plus récemment, à propos de l'appareil judiciaire (cf. Fieulaine et al., 2013). D'abord, le système d'accès au droit est critiqué par une partie de notre échantillon, qui ne l'objective pas ou avec difficultés et le reconnaît à l'envers (Kalampalikis, 2006), à travers son étrangéité, produisant de la tension dans les dynamiques de recours au droit. Ainsi, le monde étrange du droit semble, pour une partie des sujets de notre enquête, être difficilement apprivoisé par la pensée sociale. Nous confirmons ainsi que la justice procédurale est plus difficilement domesticable par le sens commun (Clémence \& Doise, op. cit.) et peut ainsi apparaître comme injuste. Ce rejet peut expliquer une partie du non-recours au droit, du moins mettre en évidence une tension toujours susceptible de produire ou d'alimenter des formes de non-connaissance, de nonréception, voire de non-demande (Warin, 2010).

Au-delà des procédures, la justice institutionnelle est jugée à l'aune de théories naïves de la justice disponibles dans le sens commun. La politique sociale de l'Etat est jugée à travers les normes d'équité et d'égalité, organisant différemment les attitudes vis-à-vis de " qui a le droit à quoi ? ». Les représentations sociales de la justice, qui se construisent en lien aux conceptions de l'ordre social (ou du désordre, cf. Fieulaine et al., 2013) et des positions vis-à-vis du rôle de l'Etat (Staerklé et al., op. cit.), donnent une signification et guident les pratiques de (non-)recours aux droits sociaux et aux aides sociales, et les formes de légitimités ou d'illégitimité données aux inégalités sociales apparaissent ici aussi déterminantes dans les rapports pratiques aux droits ( cf. Blasi \& Jost, 2006).

Plus largement, face aux expériences vécues et au constat des inégalités persistantes, les représentations sociales du système de régulation des rapports sociaux chargé d'assurer la justice pénale et sociale, peuvent ne plus s'inscrire dans l'idée de justice. Les institutions ne sont alors plus reconnues comme justes. Leur image se fragilise dans une partie des discours tenus à leur égard. L'idée et la chose ne correspondent plus (Ricœur, 2005). Ainsi la figure construite de la société instituée subirait une défamiliarisation d'avec les conceptions d'un monde juste auxquelles les sujets adhèrent, activant encore un mode d'ancrage stigmatique (Kalampalikis \& Haas, 2008). L'expérience du monde est dans ce cas vécue à travers un certain sentiment d'étrangéité, de désenchantement. Les sujets expriment alors une perte de confiance dans les institutions régulatrices des rapports sociaux, qui est signifiée à travers la réaffirmation de valeurs fondamentales, de " croyances axiologiques » 
(Boudon, 1995) et des idées-forces des droits humains en tant que représentations sociales normatives (Doise, 2001).

Cette fragilisation des représentations sociales génère de la méfiance, voire de la défiance envers les institutions, et peut entrainer une participation sociale marquée par un certaine distance avec cette société, par une intention d'en sortir, qui peut se caractériser par des pratiques religieuses, des replis individualistes, un nihilisme politique, mais également par des projets et/ou des pratiques d'autonomie collective et politique; montrant ainsi des lignes de tensions, des conflits et des compromis se cristallisant dans les rapports et les liens à la société instituée et par conséquent dans les recours aux droits. Ainsi, dans leur expérience vécue, les sujets construisent des représentations critiques de la société telle qu'elle est instituée mais toujours orientée vers une revendication performative de la justice, retranscrite dans les pratiques de recours aux droits. Par conséquent, la fragilisation des représentations sociales ne doit être comprise comme un processus négatif, mais davantage à travers son potentiel de changement social, en direction de la demande ou du recours aux droits, mais aussi potentiellement de la revendication de droits.

\section{Notes}

1. La catégorie du non-recours est d'abord apparue dans les pays anglo-saxons, en Angleterre dans les années 1950, puis aux Pays-Bas et aux Etats-Unis, dans les années 60. C'est notamment dans un objectif gestionnaire que des recherches ont alors été menées, servant à justifier la suppression de certaines aides sociales. Plus récemment, le contexte de crise économique et de restriction budgétaire donne à observer dans des pays européens, la conciliation entre volonté de favoriser la justice sociale, notamment par l'amélioration de l'accès aux droits sociaux, et un contrôle de plus en plus strict des dépenses publiques (Hamel, op. cit.).

2. Par exemple, il a été mis en évidence que le taux moyen de non-recours aux programmes sociaux se situe entre $20 \%$ et $40 \%$ dans I'OCDE (Hernanz, Malherbet, Pellizzari, 2004) et il n'est jamais inférieur à $10 \%$ dans les travaux de l'Observatoire Odenore pour la France (Warin, 2011). Le non-recours au Revenu de Solidarité Active (RSA) concerne une personne éligible au dispositif sur deux (Domingo \& Pucci, 2012).

3. Nous renvoyons le lecteur au site internet de l'Odenore qui recense largement la production scientifique sur cet objet : http://odenore.msh-alpes.fr.

4. Nous nous sommes basées sur les statistiques produites par l'association

5. 52 ans, interviewé dans une $\mathrm{BD}$, fait des démarches pour récupérer son Allocation adulte handicapé que l'administration ne lui accorde plus.

6. 58 ans, interviewé dans une $\mathrm{BD}$, ancien chef d'entreprise, revendique la reconnaissance d'une maladie professionnelle par le Régime Social des Indépendants.

7. Proche du retraitisme pointé par Robert et Faugeron (op. cit.), mais davantage dans un logique d'innaccessibilité en tant que telle, que de rejet.

8. Interviewé à ALIS, 27 ans, au RSA, sans logement, en formation.

9. Interviewé à ALIS, 33 ans, touche l'allocation chômage, hébergé dans un hébergement d'urgence. 
10. Sujet ayant des « vrais » besoins, adhérant à la valeur travail et/ou étant français.

11. Interviewé à ALIS, 59 ans, au RSA, vient d'intégrer une chambre dans une résidence sociale.

12. Interviewé à ALIS, 25 ans, au RSA, habite en squat.

13. Josiane, interviewé dans une $\mathrm{BD}$, à qui ont vient de refuser, à 52 ans, le renouvellement de son Allocation Adulte Handicapé $(\mathrm{AAH})$, exprime toute la difficulté qu'elle a à devoir la revendiquer tout en s'y sentant obligé d'un point de vue matériel : "Quand vous vous rendez compte, vous vous voyez dans la glace, que vous vous rendez compte comment vous êtes, que, et vous passez devant huit personnes presque (HESITATION), presque nue, qui vous regarde tous. Vous voyez, je veux dire. C'est pas facile. Déjà accepter à faire ça (SILENCE). Mais si je dois pour. Sinon, comment je fais pour vivre? Je veux pas me retrouver dans la rue !»

14. Cette autonomie revendiquée par certains sujets, notamment à ALIS, est moins attribuables au capital culturel des sujets - bien que celui-ci ait son importance - qu'au capital expérientiel acquis à travers la plus ou moins grande socialisation juridique. Ainsi, des sujets affirment, comme Thierry, (39 ans, au RSA, sans logement), que certaines pratiques de droit sont « inscrites dans les gênes ». Ou encore, Nasser (39 ans, sans logement, recourt à l'hébergement en foyer) qui explique que durant son enfance il a "été assisté pendant plusieurs années » ce qui lui permet, paradoxalement, de ne s'être " jamais senti comme un assisté ».

\section{Bibliographie}

- Amsterdam, A. G., Bruner, J. (2000). Minding the Law. Cambridge: Harvard University Press.

- Abraham, C., \& Sheeran, P. (2003). Acting on intentions: The role of anticipated regret. British Journal of Social Psychology, 42(4), 495-511.

- Andrade, C. (2002). The Economics of Welfare Participation and welfare stigma : A Review. Public Finance and Management, 2(2), 294-333.

- Azzi, A. E., Klein, O. (1998). Psychologie sociale et relations intergroupes. Paris : Dunod.

- Baraquin, Y. (1973). Les justiciables face à la justice civile (approche qualitative). Paris : C.R.E.D.O.C.

- Blasi, G., \& Jost, J. T. (2006). System justification theory and research: Implications for law, legal advocacy, and social justice. California Law Review, 1119-1168.

- Berrat, B. (2011). Le non-recours aux droits et dispositifs liés au handicap. In M. Jaeger (Ed.). Usagers ou citoyens? De l'usage des catégories en action sociale et médico-sociale. Paris : Dunod. pp. 137-155.

- Boltanski, L. (1990). L'amour et la Justice comme compétence. Paris : Métailié.

- Boltanski, L., Chiapello, E. (1999/2011). Le nouvel esprit du capitalisme. Paris : Gallimard.

- Boudon, R. (1995). Le juste et le vrai. Paris, Fayard.

- Castel, R. (1995). Les pièges de l'exclusion, Lien social et politiques - RIAC, 34, 13-21.

- Castel, R. (2003). Des individus sans supports. In V. Châtel \& M.-H. Soulet (Eds.), Agir en situation de vulnérabilité. Saint-Nicolas (Québec) : Presses de l'Université de Laval.

- Castel, R. (2009). La montée des incertitudes. Travail, protection, statut de l'individu. Paris: Le Seuil.

- Cattell, R.B. (1966). The scree test for the number of factors. Multivariate behavioural research 1, 246-276. 
- Catrice-Lorey, A. (1976). Inégalités d'accès aux systèmes de protection sociale et pauvreté culturelle. Revue Française des Affaires Sociales, 30(4),127-137.

- Chauchat, H. (1995). L'enquête en psycho-sociologie. Paris : PUF.

- Chauveaud, C., Demezière, D., Dethyre, R., Djoulden, M., Féré, C., Gajdos, T., Laumet, D., Levy, J., Mazet, P., Okbani, N., Revil, H., Saillard, Y, Warin, P. (2012). L'envers de la "fraude sociale ». Le scandale du non-recours aux droits sociaux. Paris, La Découverte.

- Clémence A, Doise W. (1995). La représentation sociale de la justice: Une approche des droits dans la pensée ordinaire. L'Année Sociologique 52, pp. 369-398.

- Clémence, A., Doise, W., de Rosa, A.M., Gonzalez, L. (1995). La représentation sociale des Droits de I'Homme. Journal International de Psychologie, 30, 181-212.

- Clémence, A., Egloff, M., Gardiol, N., Gobet, P. (1994). Solidarités sociales en Suisse. Lausanne : Réalités sociales.

- Doise, W. (2001). Droits de l'homme et force des idées. Paris : PUF.

- Doise, W. (2007). Psychologie sociale et norme de justice. Bulletin de psychologie, 60 (5), 491, 389-396.

- Doise, W. (2009). Discriminations sociales et droits universels. Grenoble : PUG.

- Domingo, P., Pucci, M. (2013). Les vecteurs du non-recours au revenu de solidarité active du point de vue de l'usager. Informations sociales, 4 (178), 72-80.

- Dubouchet, J. (2004). Les représentations sociales de la justice pénale : Retour sur un chantier abandonné. Déviance \& Société, 28, pp. 179-194.

- Ewick, P., Silbey, S. (1998). The Commonplace of Law. Stories of Everyday Life. Chicago, University of Chicago Press. (Deux extraits du chapitre III ont été publiés sous le titre "La construction sociale de la légalité » dans Terrains et travaux, 6(1), 2004, 112-138.)

- Felstiner, W.L.F., Abel, R.L., Sarat, A. (1981). The Emergence and Transformation of Disputes: Naming, Blaming, Claiming. Law \& Society Review, (15)3/4, 631-654.

- Felstiner, W. L., Abel, R. L., \& Sarat, A. (1991). L'émergence et la transformation des litiges: réaliser, reprocher, réclamer. Politix, 4(16), 41-54.

- Fieulaine, N. (2007). Temps de l'urgence, temps du projet : La rencontre des temporalités dans le recours aux soins et à I'aide sociale en situation de précarité. Dossiers de la MRIE 15, 41-45.

- Fieulaine, N. \& Apostolidis, T. (in press). Precariousness as a time horizon: How poverty and social insecurity shape individuals' time perspectives. In M. Stolarski, N. Fieulaine \& W. VanBeek (eds.) Time Perspective ; Theory, Research and Applications. New York, Springer.

- Fieulaine, N., Kalampalikis, N., Haas, V. (2009). Usages du droit et représentations de la justice. Le (non) recours au droit. Rapport de recherche, Mission de Recherche Droit \& Justice \& GRePS-UL2.

- Fieulaine, N., Kalampalikis, N., Haas, V. \& Béal, A. (2013). Les représentations sociales de I'appareil judiciaire: principes organisateurs, expériences et postures idéologiques. Revue Internationale de Psychologie Sociale, 26(1), 35-59.

- Gurvitch, G. (1935). L'expérience juridique et la philosophie pluraliste du droit. Paris : Pedone.

- Hamel, M.-P. (2013). Politiques d'accès aux droits sociaux : une approche comparée dans trois pays européens. Informations sociales, 4(178), 24-32. 
- Hermitte, M.-A. (1999). Le droit est un autre monde. Enquête, 7, url : <http://enquete. revues.org/1553>.

- Hernanz, V., Malherbet, F., Pellizzari, M. (2004). Take-up of Welfare Benefits in OECD Contruies : A Review of the Evidence. Paris : OECD.

- Moscovici, S., Hewstone. M. (1984). De la science au sens commun ; in Serge Moscovici (éd.), Psychologie sociale. Paris, P.U.F. 539-566.

- Hunout, P. (1987). La psychologie sociale des décisions de justice : une discipline en émergence. Déviance et société. 11(3), 271-292.

- Israël, L. (2009). L'arme du droit. Paris : Les Presses de Sciences Po.

- Jodelet, D. (éd.) (1989). Les représentations sociales. Paris, P.U.F.

- Jodelet, D. (2006). Place de l'expérience vécue dans les processus de formation des représentations sociales. In V. Haas (Ed.), Les savoirs du quotidien. Transmissions, appropriations, représentations. Rennes, PUR. pp. 235-255.

- Jodelet, D. (2013). La Rencontre des Savoirs. Papers on Social Representations, (22)9, pp. 1-20.

- Kalampalikis, N. (2002). Des noms et des représentations. Les Cahiers Internationaux de Psychologie Sociale, 53(1), 21-30.

- Kalampalikis, N. (2006). Affronter la complexité : représentations et croyances. In V. Haas (Ed.), Savoirs du quotidien. Transmissions, Appropriations, Représentations. Rennes : PUR, pp. 229-237.

- Kalampalikis, N. (2007). Les Grecs et le mythe d'Alexandre. Etude psychosociale d'un conflit symbolique à propos de la Macédoine. Paris : L'Harmattan.

- Kalampalikis, N., Haas, V. (2008). More than a theory : a new map of social though. Journal for Theory of Social Behaviour, 38(4), 449-459.

- Kourilsky-Augeven C. (1990). Les processus de socialisation juridique : la formation des connaissances et de représentations du droit avant l'âge adulte, Rapport intermédiaire. Paris: CNRS.

- Languin, N., Widmer, É. D., \& Kellerhals, J. (2004). Les représentations sociales de la justice pénale: une trilogie. Déviance et société, 28(2), 159-178.

- Lewin, K. (1942). Time Perspective and Morale. In G. Watson (Ed.), Civilian Morale (pp. 4870). Boston : Houghton Mifflin.

- Lerner, M. J. (1980). The belief in a just world. A fundamental delusion. New York: Plenum Press.

- Markovà, I. (2007). Dialogicité et représentations sociales. Paris : PUF.

- Mazet, P. (2010). La non-demande de droits : prêtons l'oreille à I'inaudible, La Vie des idées,

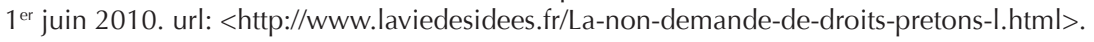

- Miller, R. E., \& Sarat, A. (1980). Grievances, claims, and disputes: Assessing the adversary culture. Law and Society Review, 525-566.

- Moscovici, S. (1961/1976). La psychanalyse, son image et son public. Paris : PUF.

- Moscovici, S. (1994). Les formes élémentaires de I'altruisme. In S. Moscovici, Psychologie sociale des relations à autrui, Paris : Nathan. 2000, 71-86.

- Moscovici, S. (1996). Communications et représentations sociales paradoxales. In J.-C. Abric (éd.). Exclusion sociale, insertion et prévention. Saint-Agne, Erès, 19-22.

- Moscovici, S. (2012). Raison et cultures. Paris : Editions EHESS. 
- Moscovici, S. (2013). Le scandale de la pensée sociale. Paris, Ed. de l'Ehess.

- Pélisse, J. (2003). Consciences du temps et consciences du droit chez des salariés à 35 heures. Droit et société, 1(53), 163-186.

- Pélisse, J. (2005). A-t-on conscience du droit ? Autour des legal consciousness studies. Genèses, 59, 114-130.

- Coates, D., Penrod, S. (1980). Social Psychology and the Emergence of Disputes. Law \& Society Review, (15)3/4, Special Issue on Dispute Processing and Civil Litigation. pp. 655680.

- Percheron, A. (1991). Représentations de la loi et de la justice chez les Français de 16 à 21 ans. Droit et Société, 19, pp. 377-388.

- Ricoeur, P. (2005). Le juste, la justice et son échec. Paris : L'Herne.

- Robert, P., Faugeron, C. (1978). La justice et son public. Les représentations sociales du système pénal. Genève, Masson.

- Roux, P., Clémence, A. (1999). Schème de raisonnement dans la justice sociale ; in J.-L. Beauvois, N. Dubois, W., Doise, La construction sociale de la personne. Grenoble : PUG, pp. 103-111.

- Sarat, A. (1990). "... The Law Is All Over» : Power, Resistance and the Legal Consciousness of the Welfare Poor. Yale Journal of Law and Humanites, 2, 343-379.

- Scharnitzky, P., Kalampalikis, N. (2007). Analyse lexicale des sources d'influence dans les jurys d'assises. Bulletin de psychologie, 60 (5)/491. 425-431.

- Simmel, G. (1995). Le conflit. Paris : Circé.

- Staerklé, C., Delay, C., Gianettoni, L., Roux, P. (2007). Qui a droit à quoi ? Représentations et légitimation de l'ordre social. Grenoble : PUG.

- Stuber, J., \& Schlesinger, M. (2006). Sources of stigma for means-tested government programs. Social Science \& Medicine, 63(4), 933-945.

- Van Oorschot, W., Math, A. (1996). La question du non-recours aux prestations familiales. Recherches \& Prévisions, 43, 5-17.

-Vrancken D. (2010a), Le Nouvel Ordre protectionnel. De la protection sociale à la sollicitude publique, Lyon, Éditions Parangon.

- Wacquant, L. (2007). Paria Urbains. Ghetto, banlieues, État. Paris : La Découverte.

- Warin, P. (2007). L'accès aux droits sociaux. Grenoble : PUG.

- Warin, P. (2010). "Le non-recours : définition et typologies ». Document de travail. №1. Observatoire des non-recours aux droits et services (ODENORE).

- Warin, P. (2010a). Ciblage de la protection sociale et production d'une société de frontières. SociologieS [En ligne], mis en ligne le 27 décembre 2010, consulté le 02 mai 2014. URL: http://sociologies.revues.org/3338.

- Warin, P. (2010b). Les politiques publiques face à la non-demande sociale. In Borraz O., Guiraudon, V. (Eds.). Politiques publiques, t. II, Changer la société. Paris, Presses de Sciences Po, pp. 287-312.

- Warin, P. (2011). Le non-recours par désaccord. Welfare stigma et catégorie du non-recours. In M. Jaeger (Ed.). Usagers ou citoyens ? De l'usage des catégories en action sociale et médicosociale. Paris : Dunod, pp. 117-136. 